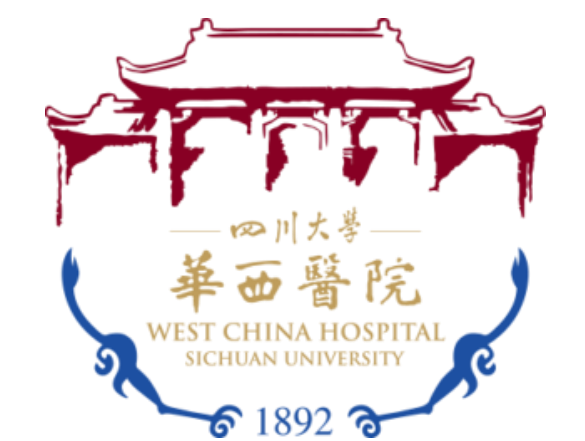

\title{
Pork belly : a novel training phantom for skill of hydro-dissection in a transversus abdominis plane block in novice anesthesia residents
}

\author{
Qi Li, M.D., Li-Qun Fang, M.D., \\ Department of Anesthesiology, West China Hospital \\ Sichuan University, Chengdu, Sichuan, China.
}

Background and Goal of Study:

Ultrasound(US)-guided transversus abdominis plane (TAP) block becomes a popular analgesic technique for lower abdominal surgeries. A skill of hydro-dissection of TAP with local anesthetics was critical for a successful TAP block. A blue phantom is useful for practicing hand-eye coordination, but not for the skill of hydrodissection. Pork belly has a similar three-layer muscular structure as that in human beings. We hypothesize that, used as a training phantom, pork belly could improve skill of hydrodissection of a TAP block in novice anesthesia residents.

\section{Materials and Methods:}

Sixteen PGY-1 anesthesia residents without USguided TAP block experience were randomized into two groups: the control group (C group) and the pork belly (PB group), each of eight. The training course consisted of two modules: a knowledge module and a second practice module. PowerPoint slides and videos were used to show (1) mechanism of sonography, (2) anatomy and sonoanatomy of TAP block, (3) and skills of hydro-dissection and image optimization in the knowledge module in both groups. In practice module, a blue phantom was used for practicing hand-eye coordination in the $\mathrm{C}$ group. In the $\mathrm{PB}$ group, a $20 \mathrm{~cm} \times 30 \mathrm{~cm}$ pork belly was used for practicing hand-eye coordination and hydrodissection. The practice module would not end until all trainees reached a mastery level. Skills of hand-eye coordination and hydro-dissection were tested respectively in a $20 \mathrm{~cm} \times 30 \mathrm{~cm}$ pork belly in both groups. Rates of mastery of hand-eye coordination and hydro-dissection were compared between the two groups using chi-square test. A $\mathrm{P}<0.05$ was considered as statistically significant.

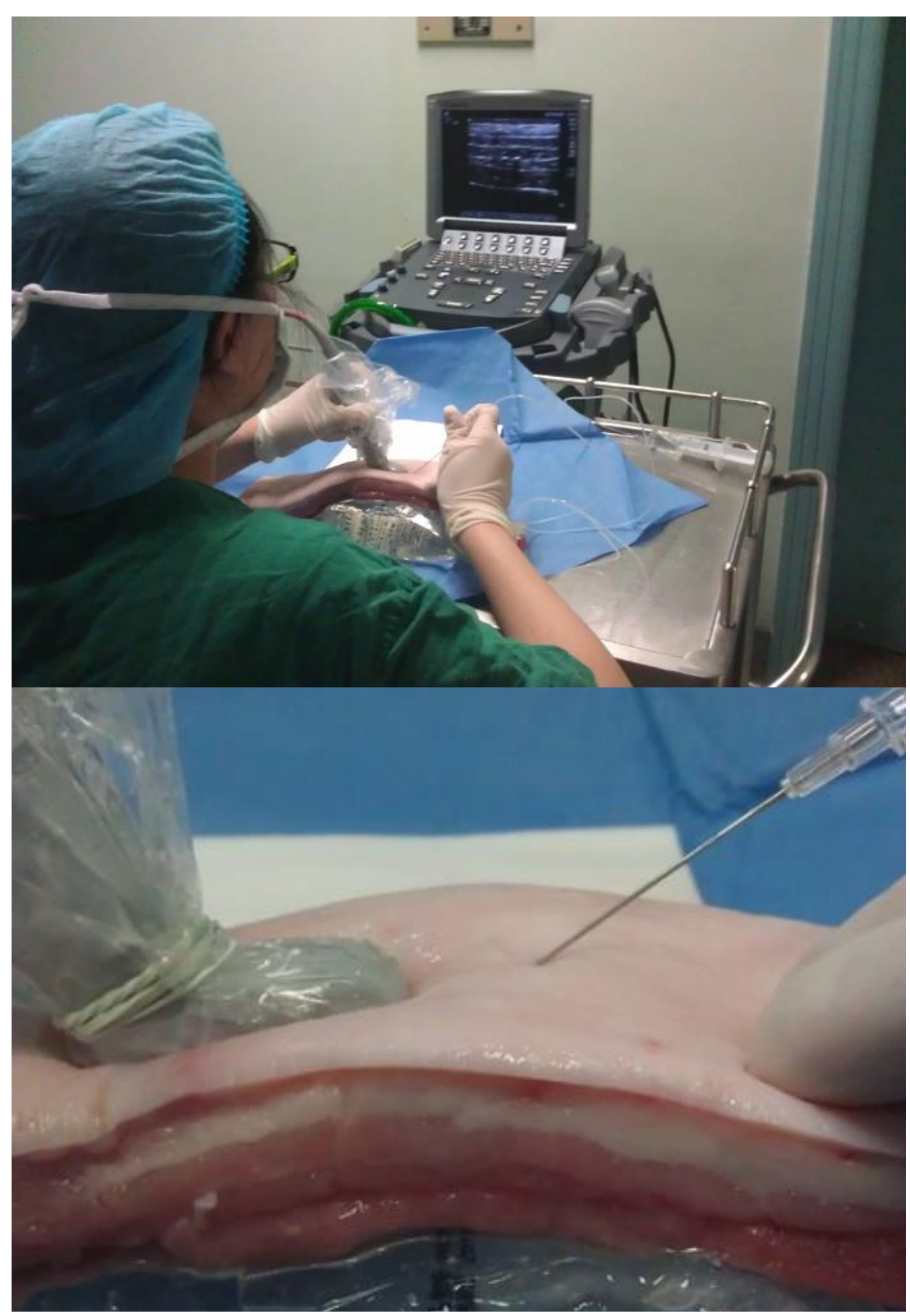

Results and Discussion:

Better mastery rate of hydro-dissection was observed in the PB group. An average of $7.8 \pm 2.4$ trials was needed to reach a mastery level of hydro-dissection skill in the PB group. With a three-layer muscular structure, pork belly could be a good phantom to simulate anterior abdominal wall in human beings, which is useful for novice anesthesia residents to practice skill of hydro-dissection in a TAP block before their clinical practice.

\section{Conclusion(s):}

Used as a training phantom, pork belly could be useful for novice anesthesia residents to practice skill of hydro-dissection in a TAP block. 